\title{
Editorial Salus publica lex suprema
}

\author{
Ruediger Rau
}

Published online: 21 October 2009

(C) Springer-Verlag 2009

Dear reader,

This issue of the Journal of Public Health presents articles that cover a broad range of Public Health topics in theory and practice. They have been contributed by authors from a global scientific community; some of them document successful international collaborations. The range of topics contains issues like stillbirth, community knowledge on stroke, aspects of quality and costs of health care in hospitals, and also being touched on are questions of health equity and ethics.

Thus, the scientific readership can look forward to reading an intriguing and well-balanced selection of new studies and findings in Public Health.

The first article, written by Reime and colleagues, addresses an important socio-economic health issue: Is there a relationship between parents' unemployment and risk of stillbirth? Given the fact of high rates of unemployment in Germany as well as in other industrialized countries, the findings of this Canadian-German study are highly interesting and relevant to researchers, practitioners and decision-makers in (health) policy and prevention.

The next article describes an innovative approach to communicate health information to the public: "We style up your knowledge!" was a joint project of the Public Health service and the hairdressers' guild in the district of Wesel, located in the Lower Rhine region. In the course of a 5-year program ("Healthy Lower Rhine...against Stroke") it

\footnotetext{
R. Rau $(\triangle)$
}

District of Wesel,

Department of Health (Fachbereich Gesundheitswesen),

Muehlenstr. 9-11,

47441 Moers, Germany

e-mail: ruediger.rau@kreis-wesel.de became increasingly clear how important it is to find the right marketing measures in order to impart health information to relevant target groups. In collaboration with an economist specialized in marketing (Mr. Leifeld), the authors were able to open up several new approaches to make health information effective and affordable, approaches that otherwise would be associated with commercial products.

Overweight and obesity in children and adolescents are growing problems in Europe, with prevalence rates showing a north-south divide reaching up to almost $35 \%$ of affected children in countries like Portugal, Greece or Italy. Physical activity and sports are of major importance for the prevention of overweight and secondary disorders.

Against this background, Federico and colleagues reveal essential new evidence in Public Health research showing significant socio-economic differences with respect to physical activity in Italian children. Consequently, these findings should be considered thoroughly when health promotion is being planned and implemented in order to mitigate social inequalities in the health and lifestyles of children.

While physical activity in our societies is on the decrease, mobility through road traffic grows steadily. With her article "Risk Exposure and Health Equity" Fahlquist not only reminds us of the burden of disease and mortality due to modern road traffic accidents (a fact that seems to be ignored far too often in health policy debates), but she also introduces an ethical perspective for analyzing issues of traffic safety. On the basis of five "ethical problem areas," such as criminalization, paternalism, privacy, justice and responsibility, the author proposes to incorporate this new ethical perspective in road traffic planning. Given a number of 1 million casualties in road traffic accidents worldwide every year, this consideration seems more than worthwhile in order to save lives in the future. 
Kainzinger and colleagues present an empirical study investigating hospital length of stay and case management, an issue of crucial importance to hospitals in general. The article describes an innovative case management system, which - according to the authors - does not negatively affect the therapy sovereignty of medical doctors, while it helps to improve process efficiency and contain or even reduce costs. Socio-demographic changes, i.e., an aging society with a rise of chronic diseases on the one hand and limited health budgets and soaring costs, partially due to rapid medical progress, on the other hand, will certainly increase the pressure to take this topic into closer consideration.

The following contribution takes us to India and to the issue of costs and utilization of health care. Singh reports that the need for health care is growing rapidly and that at the same time the number of private health care institutions is increasing significantly. Yet, little is known about the use and costs in the private health care sector in India. Singh selected data sets focusing on five diagnosis groups and found that costs in private health services are substantially higher than in the public sector. However, the majority of patients utilized private health care services. The study results suggest strong socio-economic inequalities in health care utilization in India.

A Japanese health statistics expert, Emi Sato, analyzed data from the German Long-Term Care Insurance 1999-2005 and compared time trends and differences between the federal states (Bundesländer). While patients in city states seemed to prefer home-care services, people in other Bundesländer rather sought care in nursing homes. This interesting study winds up with a little surprise...despite demographic changes and an aging population, the number of people needing care seems to decline?!
The next article deals with an important pedagogical issue. Loureiro and colleagues present a Problem-Based Learning (PBL) approach and suggest that training and education of the European Public Health working force can thus be optimized, i.e., rendered more efficient. Not only thinking and analyzing in a systemic and comprehensive way is crucial to solving Public Health problems, but also practical skills, like teamwork, goal-oriented negotiation and assertiveness, are important for an effective work in this complex field.

The second to last contribution tackles a very pivotal problem in Public Health, i.e., the critical appraisal of clinical trials: How objective and neutral are interpretations of clinical trials depending on the motivation and interests of interpreting persons?

Porzsolt and colleagues introduce "Twin Assessment of Clinical Trials (TACT)" as a new evaluation instrument. Even with evidence-based assessment tools, it seems to be difficult to come to reproducible interpretations. This study reminds the scientific community to always stay prudent and cautious when dealing with results from clinical trials and interpretation of these results. Clinical trials cannot replace experience gained under "real world conditions."

Finally, Fangerau comments on the TACT study, concluding that the twin assessment approach might be useful in the future in coping with conflicts of interest, while safeguarding scientific values.

Dear reader, I hope you will enjoy reading the topical edition of the JPH, gaining new insights, ideas and suggestions that will be helpful for your work in theory and practice.

I wish you a Merry Christmas and a Happy New Year. 